\title{
Balance Right in Multiple Sclerosis (BRiMS): a feasibility randomised controlled trial of a falls prevention programme
}

\author{
H. Gunn ${ }^{*}$ DD, K. N. Stevens ${ }^{2,3}$, S. Creanor ${ }^{2,4}$, J. Andrade ${ }^{5}$, L. Paul ${ }^{6}$, L. Miller ${ }^{7}$, C. Green ${ }^{8}$, P. Ewings ${ }^{9}$, A. Barton ${ }^{10}$, \\ M. Berrow ${ }^{2}$, J. Vickery' ${ }^{2}$ B. Marshall', J. Zajicek ${ }^{11}$ and J. A. Freeman ${ }^{1}$
}

\begin{abstract}
Background: Balance, mobility impairments and falls are problematic for people with multiple sclerosis (MS). The "Balance Right in MS (BRiMS)" intervention, a 13-week home and group-based exercise and education programme, aims to improve balance and minimise falls. This study aimed to evaluate the feasibility of undertaking a multicentre randomised controlled trial and to collect the necessary data to design a definitive trial.

Methods: This randomised controlled feasibility study recruited from four United Kingdom NHS clinical neurology services. Patients $\geq 18$ years with secondary progressive MS (Expanded Disability Status Scale 4 to 7) reporting more than two falls in the preceding 6 months were recruited. Participants were block-randomised to either a manualised 13-week education and exercise programme (BRiMS) plus usual care, or usual care alone. Feasibility assessment evaluated recruitment and retention rates, adherence to group assignment and data completeness. Proposed outcomes for the definitive trial (including impact of MS, mobility, quality of life and falls) and economic data were collected at baseline, 13 and 27 weeks, and participants completed daily paper falls diaries.

Results: Fifty-six participants (mean age 59.7 years, 66\% female, median EDSS 6.0) were recruited in 5 months; 30 randomised to the intervention group. Ten (18\%) participants withdrew, 7 from the intervention group. Two additional participants were lost to follow up at the final assessment point. Completion rates were $>98 \%$ for all outcomes apart from the falls diary (return rate $62 \%$ ).

After adjusting for baseline score, mean intervention-usual care between-group differences for the potential primary outcomes at week 27 were MS Walking Scale-12v2: - 7.7 (95\% confidence interval [CI] - 17.2 to 1.8) and MS Impact Scale-29v2: physical 0.6 (Cl -7.8 to 9), psychological -0.4 (Cl -9.9 to 9). In total, 715 falls were reported, rate ratio (intervention:usual care) for falls 0.81 (0.41 to 2.26) and injurious falls 0.44 (0.41 to 2.23).
\end{abstract}

Conclusions: Procedures were practical, and retention, programme engagement and outcome completion rates satisfied a priori progression criteria. Challenges were experienced in completion and return of daily falls diaries. Refinement of methods for reporting falls is therefore required, but we consider a full trial to be feasible.

*Correspondence: hilary.gunn100@plymouth.ac.uk

${ }^{1}$ Faculty of Health, School of Health Professions, Peninsula Allied Health Centre, University of Plymouth, Derriford Road, Plymouth PL6 8BH, England

Full list of author information is available at the end of the article

(c) The Author(s). 2020 Open Access This article is licensed under a Creative Commons Attribution 4.0 International License, which permits use, sharing, adaptation, distribution and reproduction in any medium or format, as long as you give appropriate credit to the original author(s) and the source, provide a link to the Creative Commons licence, and indicate if changes were made. The images or other third party material in this article are included in the article's Creative Commons licence, unless indicated otherwise in a credit line to the material. If material is not included in the article's Creative Commons licence and your intended use is not permitted by statutory regulation or exceeds the permitted use, you will need to obtain permission directly from the copyright holder. To view a copy of this licence, visit http://creativecommons.org/licenses/by/4.0/ The Creative Commons Public Domain Dedication waiver (http://creativecommons.org/publicdomain/zero/1.0/) applies to the data made available in this article, unless otherwise stated in a credit line to the data. 
(Continued from previous page)

Trial registration: ISRCTN13587999

Date of registration: 29 September 2016

Keywords: Secondary progressive multiple sclerosis, Exercise, Self-management, Mobility, Accidental falls, Balance, Quality of life, Feasibility randomised controlled trial

\section{Key messages on feasibility}

(i) What uncertainties existed regarding feasibility? It was unknown whether the recruitment strategies and processes would be effective, and whether participants would be able to maintain engagement with the trial and the BRiMS programme. There were also uncertainties about the choice of a primary outcome for a full effectiveness trial, and the methods used for collecting prospective falls data.

(ii) What are the key findings on feasibility from this study? The trial methods were feasible and effective in recruiting and retaining participants, although some changes to the BRiMS programme were indicated to reduce attrition. Most outcome measures had satisfactory completion; however, there were challenges in the methods of collecting falls data.

(iii) What are the implications of the feasibility findings on the design of the main study? The findings indicate that a large-scale trial is feasible; however, refinement of falls reporting methods and development of BRiMS programme delivery methods are recommended prior to progressing further.

\section{Background}

Multiple sclerosis (MS) is an incurable, unpredictable but typically progressive, life-long, neurological condition, affecting approximately 100,000 people in the UK (UK) [1]. It is the most common cause of neurological disability in young adults. Although most people start with a relapsingremitting (RR) disease course, approximately two-thirds move to a progressive phase, with a steady rise in the proportion of progressive cases as the disease advances.

Within approximately 15 years of diagnosis, an estimated $50 \%$ of people are unable to walk unaided, and eventually $25 \%$ are dependent on a wheelchair [2]. An important contributor to this is impaired balance, which is reported by approximately $75 \%$ of people with MS [3]. Mobility is more compromised in those with secondary progressive MS (SPMS) compared to RR MS [4]. Our previous work suggests that falls may be an early marker of mobility deterioration associated with disease progression [5]. Rehabilitation interventions which improve balance and mobility, and therefore decrease the risk of falls, may slow this deterioration, providing a persuasive argument for ensuring this should be a clinical priority.
With only limited medical interventions available for this patient group, rehabilitation programmes are considered key to management but currently lack a robust evidence base [6].

In partnership with service users, providers of rehabilitation services, other key stakeholders (including service commissioners) and international collaborators, our ongoing research programme has systematically developed 'Balance Right in MS' (BRiMS), an innovative 13-week evidence-based, user-focused, manualised, self-management programme, designed to improve safe mobility and reduce falls for people with MS [5, 7, 8]. The programme includes personalised education and exercise and motivation components, designed to address modifiable fall risk factors, and enable self-management by use of mobility, safety and falls risk management strategies.

\section{Aim}

The overall aim of this study was to evaluate the feasibility of undertaking a multi-centre randomised controlled trial (RCT) to compare BRiMS plus usual care with usual care alone, and to collect the necessary data to design a definitive trial. The study objectives were to determine:

1. Feasibility:

- Suitability, utility and acceptability of the study procedures

- Appropriateness of eligibility criteria,

- Viability of recruitment and randomisation procedures,

- Retention rates,

- Participant engagement throughout the study,

- Adverse events.

2. Potential definitive trial outcomes:

- The selection of primary and secondary outcome measures including:

- Their characteristics and rates of completion i.e. baseline scores, distributional properties standard deviations,

- Responsiveness and to help determine the sample size for the RCT.

3. Health economics objectives:

- Estimates of resource use and related costs associated with delivery of the BRiMS intervention 


\section{Methods}

The study was undertaken according to the methods detailed in our protocol [9], which are briefly summarised below.

\section{Study design}

This was a pragmatic, mixed-methods, multi-centre, feasibility, individually randomised, group treatment RCT, with blinded outcome assessment and embedded process evaluation.

\section{Participants}

The target population was English-speaking men and women, aged $\geq 18$ years, with a confirmed diagnosis of SPMS (Expanded Disability Status Scale (EDSS) 4-7 inclusive), who reported having walking difficulties and more than two falls in last 6 months. People were excluded if they

- had ever had previous treatment with alemtuzumab;

- were within 6 months of ceasing natalizumab; or within 3 months of ceasing any other MS diseasemodifying drug;

- reported a relapse within the last month as defined: "the appearance of new symptoms, or the return of old symptoms, for a period of $24 \mathrm{~h}$ or more-in the absence of a change in core body temperature or infection") [10];

- had been referred to a falls management programme within the previous 6 months, or

- were participating in a concurrent trial.

\section{Recruitment}

The study recruited from four UK NHS clinical neurology services. Potential participants were identified through local and national advertising, adoption on to the local NIHR Clinical Research Network portfolio and via the caseload of local MS clinicians. Due to the nature of the group-based intervention and to facilitate randomisation, participants were recruited in blocks of 8-12 individuals (for full details, refer to the study protocol [9]).

\section{Study procedures}

The participant pathway is detailed in Fig. 1. Site-based research therapists screened potential participants by telephone interview. Final eligibility checking, informed consent and baseline measures were undertaken at a single face-to-face meeting at a local healthcare venue, no more than 2 weeks prior to the pre-scheduled randomisation date for each BRiMS delivery. Randomisation was undertaken via a secure web-based system by staff from the UK Clinical Research Collaboration registered Peninsula Clinical Trials Unit who were not involved in the delivery of the study. Participants were randomised in block sizes of $8-12$. Within each block, participants were individually randomised to BRiMS plus usual care (BRiMS) or usual care only on a 1:1 basis and informed of this allocation by email.

Participants were followed-up on two occasions: at 13 weeks ( \pm 1 week) and 27-weeks ( \pm 1 week) following randomisation.

\section{Staffing}

BRiMS sessions were delivered by trained treating physiotherapists [11]. Research assessments were undertaken by research physiotherapists who were aware of the study aims but were blinded to individuals' allocated group.

\section{Interventions (see Fig. 2)}

In addition to usual care, participants allocated to the BRiMS programme were asked to undertake a home exercise and falls prevention education programme. This aimed to support participants to achieve a minimum of $120 \mathrm{~min}$ of individualised, progressive, gait, balance and functional training per week, and to complete four education packages (focussing on enabling the development of falls prevention strategies and self-efficacy) over the 13 weeks. Participants were invited to attend two oneto-one sessions: an initial assessment and goal setting session at local NHS/ university physiotherapy facilities, and a home visit to explain and demonstrate use of the online resources, support the home exercise programme and to problem solve any issues. Ongoing support was provided by online resources, a paper-based manual, biweekly reviews of participants' online exercise logs by therapy staff and three, 2-h group sessions at local NHS/ university physiotherapy facilities for peer support, group exercise and interactive learning activities over the course of the programme.

Participants allocated to the usual care group continued to receive their usual clinical input. Although usual care varies across the country [12], it rarely involves regular ongoing physiotherapy intervention on either an individual or a group basis. With the exception of the study assessments, they were not asked to attend any additional visits or sessions. Data on the nature/frequency of usual care for all participants was captured via a resource use questionnaire at each assessment time-point.

\section{Sample size}

The target sample size was 60 participants across four UK sites in two regions (40 in the South West of England and 20 in Ayrshire, Scotland) to be recruited over 6 months. This would allow estimation of the overall retention rate with precision (using a 95\% confidence interval) of at least $\pm 13 \%$, improving to a $\pm 10 \%$ precision if the 27 -week follow-up rate was around $80 \%[13,14]$. Assuming a nondifferential follow-up rate of $80 \%$, this recruitment target was anticipated to provide follow-up data on a minimum of 


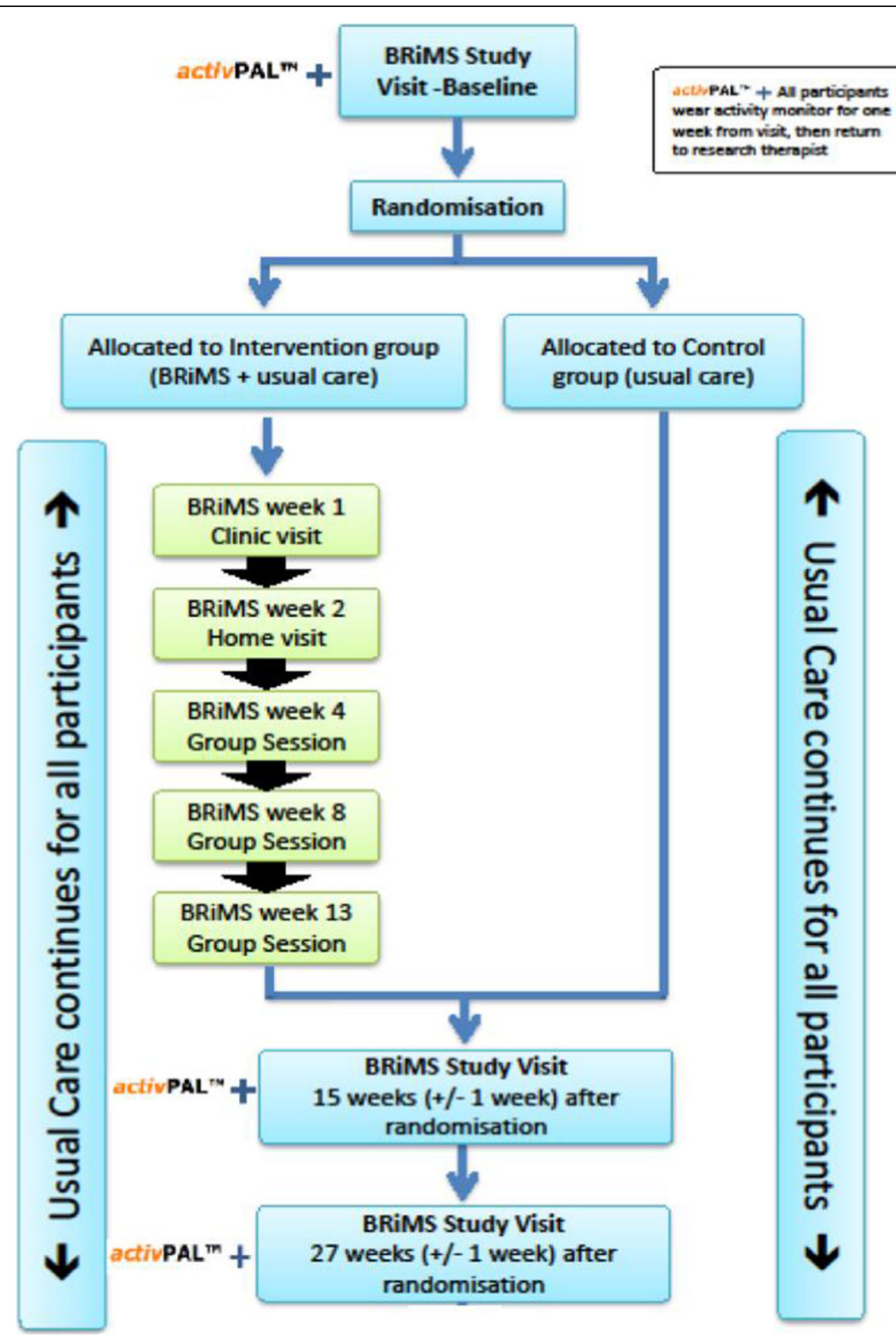

Fig. 1 Participant pathway

24 participants in each of the allocated groups, sufficient to calculate estimates of variability for the proposed outcome measures to inform indicative sample size calculations for the definitive trial.

\section{Outcomes}

Outcome measures appropriate to each of the study objectives are listed in Table 1, with further detail included in Additional file 1 . Potential primary and secondary outcome measures were chosen based on their relevance to the aims of the BRiMS programme and that there was psychometric evidence to support their use. Although the reduction of accidental falls was a key aim of BRiMS, falls rates (calculated from prospectively completed falls diaries, returned every 2 weeks) were considered a potential secondary rather than primary outcome, due to concerns about the reliability and validity of self-report falls diary data [15]. Instead a psychometrically robust mobility measure was chosen as a 


\section{BRiMS Programme}

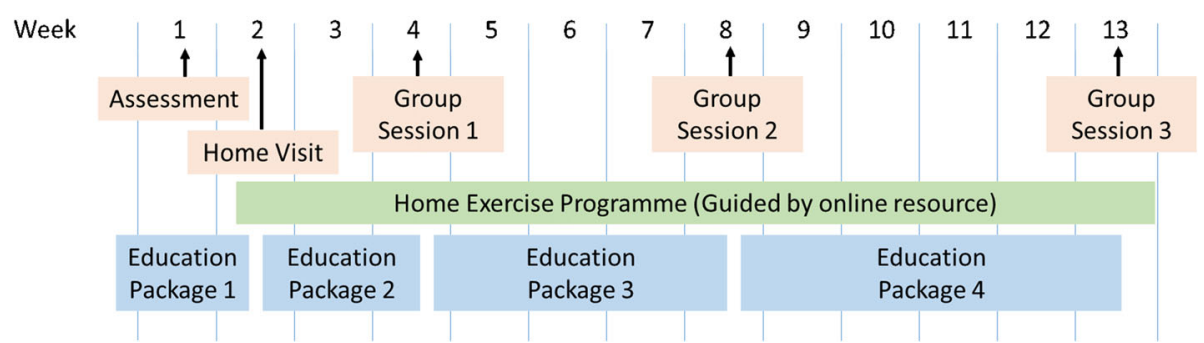

Fig. 2 BRiMS programme delivery plan. SAE: serious adverse reactions (as classified by the study principle investigator/ chief investigator (see study protocol for details) [9]. Only a sub-group of participants were invited to participate in the qualitative interview. ${ }^{* *}$ One participant did not complete baseline data for the EQ5D-5L potential primary outcome

potential primary outcome, on the basis that this was likely to reflect changes in "safe" mobility which was a key goal of the BRIMS programme"

\section{Progression criteria}

A number of progression criteria were pre-defined in discussion with the Trial Steering Committee (TSC):

1. A minimum of $80 \%$ recruitment within the planned 6-month recruitment window

2. A minimum of $80 \%$ participants randomised to BRiMS engaging with the programme (defined as attending the initial face-to-face clinic visit and home visit).

3. A minimum of $80 \%$ completion rate of at least one of the proposed primary outcome measures amongst participants attending the planned primary end-point of 27 ( \pm 1 week).

4. That the total resource estimated to conduct the definitive trial is within a level that is likely to attract funding.

\section{Data analyses}

A detailed statistical analysis plan was approved by the TSC prior to database lock. The statistical analyses were undertaken using StataSE version 14, supplemented where required by $\mathrm{R}$ [25].

Analyses were undertaken on a modified Intention To Treat (mITT) basis, with additional analysis of the falls data as outlined below, and in accordance with guidelines for pilot and feasibility trials [26]. Descriptive statistics were used to summarise patient eligibility, recruitment, allocation and retention, demographic and clinical characteristics, outcome measures and their completeness. Where appropriate, parameter estimates (e.g. between-group differences, both unadjusted and adjusted for baseline values where available) are presented with confidence intervals but no formal hypothesis testing was undertaken [26]. Outliers were identified and reported but not removed from the descriptive statistics unless otherwise stated. For the validated patient reported outcome measures, MS Walking Scale-12v2.0 [16] (MSWS-12v2), MS Impact Scale29v2.0 [16, 18, 19] (MSIS-29v2) and Falls Efficacy Scale (international) [23] (FESi), established methods for imputing missing item-level data were implemented when the minimum requirements were met [27-29]. A validated imputation method was not available for the Community Participation Indicators [24] score and so summaries for this score are based on complete data only.

Rates of falls and injurious falls were calculated per person per year, using two different methods: (a) the "mITT" analysis assumed that if a participant did not complete or return a diary entry for a particular day, they did not fall (i.e. missing values were replaced with zeroes/no fall); (b) the "Observed" analysis used only the completed diary data. The rates were compared between allocated groups using unadjusted rate ratios (intervention: usual care), with bootstrapped confidence intervals.

Mean health state values and quality adjusted lifeyears (QALY) estimates used in the health economics analysis were based on the EQ5D-3L [30] derived from the EQ-5D-5L health states and the MS-specific preference based measure, the MSIS-8D [31] (derived from participant reports for the MSIS-29) collected throughout the study. The QALY combines length and qualityof-life in a single outcome measure. Each year of life is weighted by quality-of-life during that time. Quality-oflife is represented by QALY weights on a scale from zero (equivalent to being dead) to one (perfect health). QALY weights can also be negative, representing quality-of-life thought worse than being dead. A higher number of QALYs indicates a better health outcome. NICE advice is to use the EQ5D-3L rather than the EQ5D-5L [32]; therefore, the EQ5D-5L was mapped to the EQ5D-3L using the "cross walk" technique [30]. 
Table 1 Outcome measures and data collection schedule [16-24]

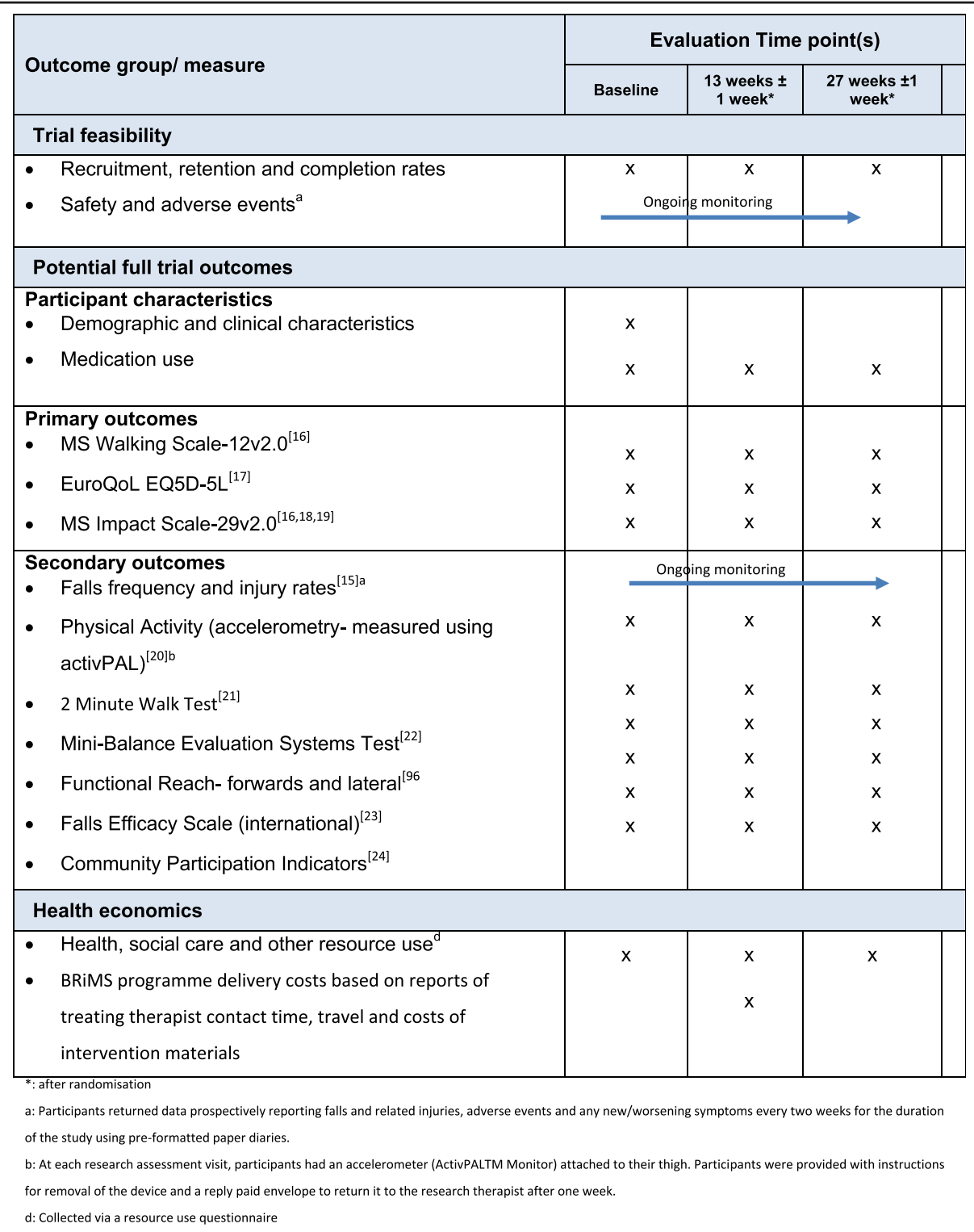

*After randomisation

aParticipants returned data prospectively reporting falls and related injuries, adverse events and any new/worsening symptoms every 2 weeks for the duration of the study using pre-formatted paper diaries

${ }^{b}$ At each research assessment visit, participants had an accelerometer (ActivPALTM Monitor) attached to their thigh. Participants were provided with instructions for removal of the device and a reply paid envelope to return it to the research therapist after 1 week

${ }^{d}$ Collected via a resource use questionnaire

\section{Results}

\section{Feasibility outcomes}

\section{Recruitment, randomisation, retention and engagement}

(Fig. 3)

Recruitment Of the 232 subjects screened over 5 months, 44 specifically declined and a further 20 were deemed ineligible on screening, leaving 56 consented participants (satisfying progression criterion 1). The main reasons for individuals declining to participate were the time commitment $(n=16 / 44,36 \%)$, and lack a computer or tablet access /poor IT literacy ( $n=14 / 44$, $32 \%)$. Despite using a range of recruitment procedures, thirty-seven $(66 \%)$ of the 56 consented participants were recruited via personal approach by research support staff or local clinicians.

Randomisation Randomisation procedures were implemented successfully, resulting in the allocation of 30 participants to the BRiMS plus usual care group, and 26 to the usual care group. 


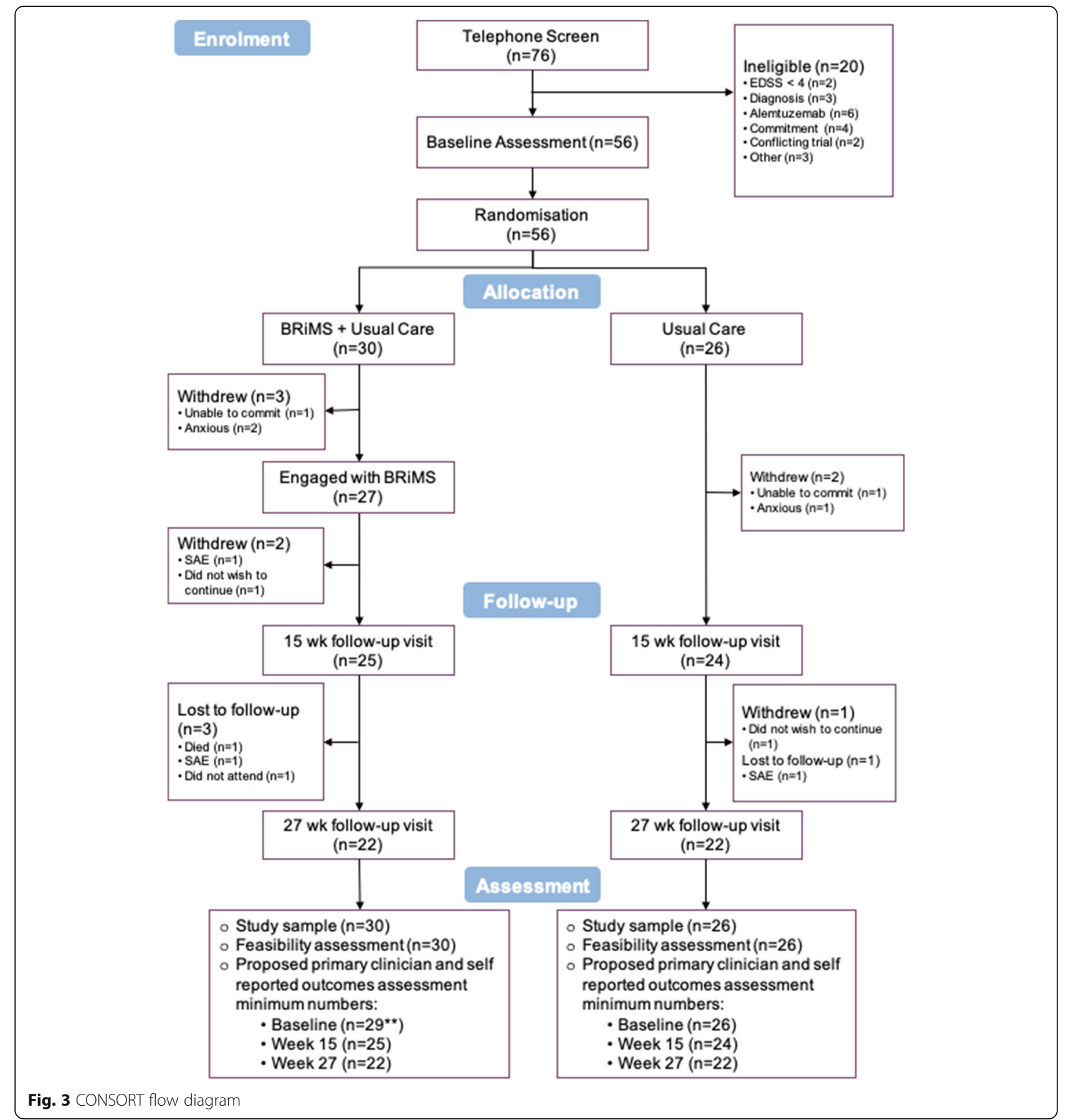

Retention The overall retention rate at week 27 was 79\% (95\% CI 66 to 88\%); there was a higher withdrawal rate in the intervention group.

Programme engagement Twenty-seven of the 30 participants $(90 \%)$ allocated to the intervention group met the criteria for engagement with the programme (satisfying progression criterion 2).
Safety and serious adverse events (SAEs) There were nine reports of SAEs from seven individuals over the study period (two usual care, five intervention). The two individuals who each reported two SAEs were in the intervention group. Despite participants all being classified with SPMS, four of the SAEs were reported to relate to MS relapses, the others to unrelated medical problems $(n=1)$ or falls with injuries requiring hospitalisation ( $n=3$; 2 intervention, 1 usual care). No SAE was assessed to be related to the BRiMS intervention. 


\section{Trial outcome objectives}

\section{Outcome measure completion rates}

At each assessment, the completion rate of each outcome measure was in excess of $98 \%$ (satisfying progresssion criterion 3). However, the overall return rate for the patient-reported falls diary was $62 \%$. There was also incomplete recording within the diaries that were returned, meaning that data were available for $58 \%$ of the expected total number of days for falls, and $41 \%$ of the expected total for injurious falls. The diary return rate was different between allocated groups (54\% BRiMS, $78 \%$ usual care).

\section{Demographic and clinical characteristics at baseline}

Table 2 details summary statistics of the participants' baseline characteristics by allocated group and for the total sample: For full details, see final study report [11]. The groups were broadly comparable at baseline, although there were differences in disease severity (EDSS), anxiety/depression and cognition (symbol digit modalities test).

\section{Outcomes}

Table 3 shows summary statistics together with the simple between-group mean differences, mean differences after adjustment for corresponding baseline score, and the indicative minimal clinically important difference (MCID), where available, for the proposed primary outcome measures. Baseline scores indicate significant mobility impairments and falls-related concern. On average, participants in the intervention group scored worse than those in the usual care group at baseline. The adjusted mean differences in the MSWS-12v2 and the MSIS-29v2 indicate that the BRiMS group improved more at 15 weeks relative to the usual care group; the adjusted between-group difference for MSWS-12v2 (physical) exceeded the MCID [21] at both 15 and 27 weeks.

Potential secondary outcomes are summarised in Additional file 2. The adjusted mean differences indicate that the BRiMS group improved more at 15 and 27 weeks relative to the usual care group in most proposed outcome measures; however, all adjusted between-group mean differences were smaller than established MCID (where available), with wide $95 \%$ confidence intervals.

$N / A$ not available, Unadjusted the mean difference between the allocated groups (BRiMS-usual care) with 95\% confidence interval for potential primary outcomes. Adjusted each participants' baseline score was subtracted from their follow-up score, and we report the mean difference between the allocated groups (BRiMS-usual care) with $95 \%$ confidence interval for potential primary outcomes

\section{Falls data}

There was substantial variation between individual falls reports over the 27-week study period (range 0-459 falls, as verified through telephone contact with the participant). One participant accounted for over half the reported falls in the usual care group; therefore, this individual was classified as an outlier and removed from the falls diary analyses presented, leaving a total of 715 falls.

\section{Falls rates (see Table 4)}

The rates of falls and injurious falls were lower in the BRiMS than the usual care group; however, the confidence intervals were wide and all included the null value (one).

Indicative sample sizes for the anticipated definitive trial The sample size calculations were undertaken for the proposed primary outcome of MSWS-12v2 at the primary endpoint of 27 weeks, to detect an improvement of 5.2 units [33] at the two-sided, 5\% significance level with $90 \%$ power. Sample sizes were also adjusted for loss to follow-up rate of $70 \%$.

As the definitive trial would be an individually randomised group treatment trial [36], the analysis would use a multi-level modelling approach, including adjustment for the baseline MSWS-12v2 score and allowing for the partially clustered data. It is assumed that participants allocated to the intervention arm would be clustered within small groups ( $~ 5$ participants), whilst participants allocated to the usual care arm would not be clustered. Therefore, the indicative sample size calculations account for a potential 'group' effect by incorporating the intra-cluster correlation (ICC) [36, 37]. Given that the intervention is standardised and that the number of BRiMS group-based sessions is small, it is assumed the ICC will be small. However to account for any potential clustering effect, the base case assumes a conservative ICC of 0.05 .

In this feasibility study, the point estimate of the SD of MSWS12-v2 at 27 weeks was 19.4 units, with one-sided $80 \%$ upper bound of 21.5 . However, a slightly inflated SD of MSWS12-v2 of 23 units is assumed, based on pooling estimates from previous relevant studies [38, 39].

Correlation estimates for MSWS12-v2 between baseline and follow-up in the SWIMS project were 0.85-0.89 [40], indicating an adjustment for this correlation should be included in the sample size calculation. The correlation in this study was 0.59 , with one-sided $80 \%$ lower bound of 0.50. Therefore, the base case assumes a correlation of 0.6.

The sample size calculations were performed in STATA using the clsampsi [37] command. The base case indicated a recruitment target of 836 participants in order to follow up 584 participants. Additional file 3 shows indicative sample sizes under a range of assumptions and shows that 
Table 2 Summary statistics of participants' clinical and demographic data at baseline

\begin{tabular}{|c|c|c|c|}
\hline Number of participants (\%) & $\begin{array}{l}\text { Usual care } \\
N=26\end{array}$ & $\begin{array}{l}\text { BRiMS } \\
N=30\end{array}$ & $\begin{array}{l}\text { Total } \\
N=56\end{array}$ \\
\hline \multicolumn{4}{|l|}{ Age (years) } \\
\hline Mean (SD) & $60.0(8.5)$ & $58.7(10.8)$ & $59.3(9.7)$ \\
\hline$[$ Min-max] & {$[46.0-81.0]$} & {$[34.0-80.0]$} & {$[34.0-81.0]$} \\
\hline \multicolumn{4}{|l|}{ Gender } \\
\hline Male & $9(34.6)$ & $10(33.3)$ & 19 (33.9) \\
\hline Female & $17(65.4)$ & $20(66.7)$ & $37(66.1)$ \\
\hline \multicolumn{4}{|l|}{ Living arrangements ${ }^{c}$} \\
\hline Alone & $9(34.6)$ & $7(23.3)$ & $16(28.6)$ \\
\hline Spouse/partner & $15(57.7)$ & $19(63.3)$ & $34(60.7)$ \\
\hline Parent/s & $1(3.8)$ & $2(6.7)$ & $3(5.4)$ \\
\hline Child/ren & $4(15.4)$ & $4(13.3)$ & $8(14.3)$ \\
\hline Other & & $1(3.3)$ & $1(1.8)$ \\
\hline \multicolumn{4}{|l|}{ Occupation status } \\
\hline Unemployed & $1(3.8)$ & $2(6.7)$ & $3(5.4)$ \\
\hline Part-time work & $4(15.4)$ & $2(6.7)$ & $6(10.7)$ \\
\hline Full-time work & $2(7.7)$ & $1(3.3)$ & $3(5.4)$ \\
\hline Age retired & $5(19.2)$ & $5(16.7)$ & $10(17.9)$ \\
\hline Medically retired & $14(53.8)$ & $19(63.3)$ & $33(58.9)$ \\
\hline \multicolumn{4}{|l|}{ EDSS $^{\mathbf{b}}$} \\
\hline Median (LQ-UQ) & $6.0(6.0-6.5)$ & $6.5(6.0-6.5)$ & $6.3(6.0-6.5)$ \\
\hline [Min-max] & {$[4.0-7.0]$} & {$[6.0-7.0]$} & {$[4.0-7.0]$} \\
\hline \multicolumn{4}{|l|}{ Cognition: SDMT ${ }^{c}$} \\
\hline $\begin{array}{l}\text { Mean (SD) } \\
{[\text { Min-max] }}\end{array}$ & $\begin{array}{l}44.5(15.1) \\
{[7.0-77.0]}\end{array}$ & $\begin{array}{l}39.1(10.9) \\
{[20.0-60.0]}\end{array}$ & $\begin{array}{l}41.6(13.2) \\
{[7.0-77.0]}\end{array}$ \\
\hline \multicolumn{4}{|c|}{ Incontinence (previous 4 weeks) } \\
\hline Not at all & $14(53.8)$ & $13(43.3)$ & $27(48.2)$ \\
\hline Once & $1(3.8)$ & $3(10)$ & $4(7.1)$ \\
\hline 2 to 4 times & $3(11.5)$ & $7(23.3)$ & $10(17.9)$ \\
\hline$>$ Weekly & $5(19.2)$ & $3(10)$ & $8(14.3)$ \\
\hline Daily & $3(11.5)$ & $4(13.3)$ & $7(12.5)$ \\
\hline \multicolumn{4}{|l|}{ Three-month fall history } \\
\hline Not fallen ${ }^{a}$ & & $1(3.3)$ & $1(1.8)$ \\
\hline Twice & $7(26.9)$ & $5(16.7)$ & $12(21.4)$ \\
\hline 3-5 times & $11(42.3)$ & $13(43.3)$ & $24(42.9)$ \\
\hline More often & $8(30.8)$ & $11(36.7)$ & 19 (33.9) \\
\hline \multicolumn{4}{|l|}{ Indoor walking aids ${ }^{c}$} \\
\hline 1 stick/crutch & $9(34.6)$ & $13(43.3)$ & $22(39.3)$ \\
\hline 2 sticks/crutches & $5(19.2)$ & $4(13.3)$ & $9(16.1)$ \\
\hline Walker/frame & $8(30.8)$ & $12(40)$ & $20(35.7)$ \\
\hline Wheelchair & $4(15.4)$ & $4(13.3)$ & $8(14.3)$ \\
\hline \multicolumn{4}{|l|}{ Outdoor walking aids ${ }^{c}$} \\
\hline 1 stick/crutch & $17(65.4)$ & $18(60)$ & $35(62.5)$ \\
\hline 2 sticks/crutches & $10(38.5)$ & $7(23.3)$ & $17(30.4)$ \\
\hline Walker/frame & $9(34.6)$ & $14(46.7)$ & $23(41.1)$ \\
\hline
\end{tabular}


Table 2 Summary statistics of participants' clinical and demographic data at baseline (Continued)

\begin{tabular}{llll}
\hline Number of participants (\%) & Usual care & BRiMS & Total \\
& $\mathbf{N = \mathbf { 2 6 }}$ & $\mathbf{N = 3 0}$ & $\mathbf{N}=\mathbf{5 6}$ \\
\hline Wheelchair & $12(46.2)$ & & $27(48.2)$ \\
Number of medications & & $5(2-7)$ & $4(2-7)$ \\
Median (LQ-UQ) & $4(2-7)$ & {$[0-17]$} & [0-17] \\
[Min-Max] & {$[0-10]$} & & $3(5.4)$ \\
Current co-morbidities ${ }^{c}$ & & $2(6.7)$ & $2(3.6)$ \\
COPD/asthma & $1(3.8)$ & $1(3.3)$ & $11(19.6)$ \\
Coronary heart disease/hypertension & $1(3.8)$ & $7(23.3)$ & $2(3.6)$ \\
Depression/anxiety & $4(15.4)$ & $1(3.3)$ & $4(7.1)$ \\
Diabetes & $1(3.8)$ & $1(3.3)$ & $9(16.1)$ \\
Migraine & $3(11.5)$ & $6(20)$ & $7(12.5)$ \\
Osteoarthritis & $3(11.5)$ & $2(6.7)$ & $21(37.5)$ \\
Osteoporosis & $5(19.2)$ & $11(36.7)$ & $2(3.6)$ \\
Other & $10(38.5)$ & $1(3.3)$ & \\
Other neurological condition & $1(3.8)$ & & \\
\hline
\end{tabular}

${ }^{a}$ There was no option for participants to report falling once

${ }^{\mathrm{b}}$ Expanded disability status scale; SDMT symbol digit modalities test

c Participants could enter in multiple options; therefore percentages may not add up to 100

the main drivers of the total sample size required are the standard deviation and the correlation between baseline and follow-up of MSWS-12v2 scores.

\section{Health economics analysis}

Methods used for economic analysis proved practical and feasible. The health economics resource use questionnaires and therapist contact sheets had completion rates greater than $98 \%$ for all those who attended assessments.

The mean cost per person for delivery of the BRiMS programme was $£ 323$. This is based on data collected on staff time by type of contact and staff type (NHS Band 7), collected within-study, aligned with published unit cost data; a mean of one clinic visit, one home visit, 5.26 online contacts and three group contacts. See the full project report [11] for further detail.

Detailed data on resource use is included in Additional file 4. Participants reported relatively modest levels of resource use over the study period, mostly focussed around items of primary and secondary care. There was little medication use reported by participants, which aligned with the study inclusion criteria, and was consistent with expectations as all participants had progressive MS. Estimated medication costs were associated with one person in each group reporting use of disease modifying therapy over the 27-week follow-up (for 25 weeks in the usual care group, and 6 weeks in the intervention group).

There was consistent reporting of informal care provision, with the reported mean hours per week similar across groups (24-25 h per week), estimated at a weekly cost of approximately $£ 445$ per participant; this being a relatively large cost component, currently provided via unpaid informal care inputs. Data on time off work by friends/relatives to support the participant was also captured, with no reports in the BRiMS group, and one participant in the usual care group reporting 13 days (mean of 0.59 days/participant in the usual care group).

As reported in detail elsewhere [11], there was some redundancy in the questionnaire items (i.e. no or minimal reports of resource use), which suggests a potential to reduce the questionnaire length in a future definitive trial.

\section{Health state values (EQ-5D, MSIS-8D) and quality adjusted life-years (QALYs)}

The data collection to inform assessment of health state values was effective, with low levels of data loss. Table 5 summarises the estimated health state values and QALY estimates.

\section{Discussion}

The results from this feasibility study inform the design of a future definitive randomised controlled trial of this exercise and education programme to improve safe mobility and reduce falls in people with progressive MS.

\section{Feasibility}

Our pre-specified thresholds for recruitment, retention and data collection were satisfied [9]. Significant variability was identified in recruitment rates depending on the approach used. Previous studies have emphasised the need for a multi-faceted recruitment strategy [41]; 
Table 3 Summary statistics, mean, standard deviation (SD) and range, and between-group mean differences of the potential primary outcome measures

\begin{tabular}{|c|c|c|c|c|c|c|c|c|}
\hline & \multirow[t]{2}{*}{$\begin{array}{l}\text { Time } \\
\text { point }\end{array}$} & \multicolumn{2}{|c|}{ Usual care } & \multicolumn{2}{|c|}{ BRiMS } & \multicolumn{2}{|c|}{$\begin{array}{l}\text { Difference between allocated groups } \\
\text { (BRiMS-usual care) } \\
\text { Mean }(95 \% \mathrm{Cl})\end{array}$} & \multirow{2}{*}{$\begin{array}{l}\text { Minimal clinically } \\
\text { important difference } \\
\text { (MCID), where } \\
\text { available }\end{array}$} \\
\hline & & $\mathrm{N}$ & $\begin{array}{l}\text { Mean (SD) } \\
{[\text { Min-max] }}\end{array}$ & $\mathrm{N}$ & $\begin{array}{l}\text { Mean (SD) } \\
\text { [Min-max] }\end{array}$ & Unadjusted & Adjusted & \\
\hline \multirow{6}{*}{$\begin{array}{l}\text { MSWS-12v2a } \\
\text { (range 0-100) }\end{array}$} & \multirow[t]{2}{*}{ Baseline } & \multirow[t]{2}{*}{26} & $79.6(14.4)$ & \multirow[t]{2}{*}{30} & $84.2(16.2)$ & & & \multirow{6}{*}{$\begin{array}{l}\text { Between } 4.0 \text { and } 6.0 \\
\text { [33] }\end{array}$} \\
\hline & & & [52.0-100.0] & & [45.0-100.0] & & & \\
\hline & \multirow{2}{*}{$\begin{array}{l}\text { Week } \\
15\end{array}$} & \multirow[t]{2}{*}{24} & $79.8(13.9)$ & \multirow[t]{2}{*}{25} & $75.6(19.4)$ & \multirow[t]{2}{*}{$-4.2(-14$ to 5.5$)$} & \multirow{2}{*}{$\begin{array}{l}-10.6 \text { (- } 18.9 \text { to } \\
2.2)\end{array}$} & \\
\hline & & & {$[48.0-100.0]$} & & [33.0-100.0] & & & \\
\hline & \multirow{2}{*}{$\begin{array}{l}\text { Week } \\
27\end{array}$} & \multirow[t]{2}{*}{22} & $79.5(21.9)$ & \multirow[t]{2}{*}{22} & $75.4(16.8)$ & \multirow[t]{2}{*}{$-4.0(-15.9$ to 7.8$)$} & \multirow[t]{2}{*}{$-7.7(-17.2$ to 1.8$)$} & \\
\hline & & & [21.0-100.0] & & {$[40.0-100.0]$} & & & \\
\hline \multirow{6}{*}{$\begin{array}{l}\text { EQ5D-3L } \\
\text { (crosswalk) }\end{array}$} & \multirow[t]{2}{*}{ Baseline } & \multirow[t]{2}{*}{26} & $0.58(0.16)$ & \multirow[t]{2}{*}{29} & $0.54(0.17)$ & & & \multirow[t]{6}{*}{$0.05-0.08[34]$} \\
\hline & & & {$[0.04-0.77]$} & & $\begin{array}{l}{[-0.04-} \\
0.88]\end{array}$ & & & \\
\hline & \multirow{2}{*}{$\begin{array}{l}\text { Week } \\
15\end{array}$} & \multirow[t]{2}{*}{24} & $0.60(0.18)$ & \multirow[t]{2}{*}{25} & $0.59(0.17)$ & \multirow{2}{*}{$\begin{array}{l}-0.01(-0.11 \text { to } \\
0.09)\end{array}$} & \multirow[t]{2}{*}{$0.03(-0.07$ to 0.14$)$} & \\
\hline & & & {$[0.20-0.91]$} & & $\begin{array}{l}{[-0.01-} \\
0.88]\end{array}$ & & & \\
\hline & \multirow{2}{*}{$\begin{array}{l}\text { Week } \\
27\end{array}$} & \multirow[t]{2}{*}{22} & $0.59(0.25)$ & 22 & $0.57(0.11)$ & $-0.02(-0.13$ to & $0.02(-0.09$ to 0.14$)$ & \\
\hline & & & $\begin{array}{l}{[-0.13-} \\
0.91]\end{array}$ & & {$[0.30-0.77]$} & & & \\
\hline MSIS-29v2 ${ }^{\mathrm{a}}$ & Baseline & 26 & $64.2(21.7)$ & 30 & $64.8(16.4)$ & & & $8.0[35]$ \\
\hline $\begin{array}{l}\text { (physical) } \\
\text { (range 0-100) }\end{array}$ & & & {$[25.0-97.0]$} & & [32.0-93.0] & & & \\
\hline & Week & 24 & $59.4(23)$ & 25 & $54.8(19.5)$ & $-4.6(-16.8$ to 7.7$)$ & $-4.9(-13.2$ to 3.5$)$ & \\
\hline & & & [13.0-98] & & [13-92] & & & \\
\hline & Week & 22 & $59.0(24.9)$ & 22 & $57.9(15.2)$ & $-1.2(-13.7$ to 11.4$)$ & 0.6 (- 7.8 to 9$)$ & \\
\hline & 27 & & [0.0.-92] & & [27-88] & & & \\
\hline MSIS-29v2 ${ }^{\mathrm{a}}$ & Baseline & 26 & $45.1(29.7)$ & 30 & $50.4(22.8)$ & & & N/A \\
\hline $\begin{array}{l}\text { (psychological) } \\
\text { (range 0-100) }\end{array}$ & & & {$[0.0-85]$} & & [4.0-96] & & & \\
\hline & Week & 24 & $43.3(26.8)$ & 25 & 43.7 (19) & 0.5 (- 12.8 to 13.8$)$ & $-5.0(-15.5$ to 5.5$)$ & \\
\hline & 15 & & [0-89] & & {$[0.0-70.0]$} & & & \\
\hline & Week & 22 & $40.0(26.8)$ & 22 & $43.3(22.6)$ & $3.3(-11.8$ to 18.4$)$ & -0.4 ( -9.9 to 9$)$ & \\
\hline & & & [0.0-93] & & {$[7.0-81]$} & & & \\
\hline
\end{tabular}

MSWS MS walking scale, MSIS MS impact scale

${ }^{\mathrm{a}}$ Decrease in score indicates improvement

${ }^{\mathrm{b}}$ Increase in score indicates improvement

Table 4 Falls and injurious falls rates (per person per year)

\begin{tabular}{|c|c|c|c|c|}
\hline & \multicolumn{2}{|c|}{ Observed } & \multicolumn{2}{|l|}{ ITT } \\
\hline & $\begin{array}{l}\text { BRiMS } \\
(N=26)\end{array}$ & $\begin{array}{l}\text { Usual Care } \\
(N=21)\end{array}$ & $\begin{array}{l}\text { BRiMS } \\
(N=30)\end{array}$ & $\begin{array}{l}\text { Usual care } \\
(N=25)\end{array}$ \\
\hline \multicolumn{5}{|c|}{ Falls (rate per person per year) } \\
\hline Rate & 38.1 & 39.1 & 21.9 & 27.0 \\
\hline Rate ratio $(95 \% \mathrm{Cl})^{\mathrm{a}}$ & \multicolumn{2}{|c|}{0.97 (0.40 to 2.22$)$} & \multicolumn{2}{|c|}{0.81 (0.41 to 2.26$)$} \\
\hline \multicolumn{5}{|c|}{ Injurious falls (rate per person per year) } \\
\hline Rate & 3.8 & 7.1 & 2.2 & 4.9 \\
\hline Rate ratio $(95 \% \mathrm{Cl})^{\mathrm{a}}$ & \multicolumn{2}{|c|}{$0.53(0.40$ to 2.21$)$} & \multicolumn{2}{|c|}{0.44 (0.41 to 2.23$)$} \\
\hline
\end{tabular}

Bootstrapped $95 \%$ confidence intervals (Cl) however, our results highlight the importance of a personal approach by clinicians or research staff.

Overall loss to follow-up was within the $20 \%$ anticipated. There was a discrepancy between retention rates in the two arms of the study, and in particular, the dropout rate in the intervention group was higher than anticipated. In comparison, a review of 26 exercise intervention studies reported combined dropout rates of $15 \%$ and $16 \%$ for intervention and usual care groups respectively [42]. We hypothesise that our higher dropout rate in the intervention group may be associated with expectations of the BRiMS programme. Further exploration of this aspect is required, supporting the notion that feasibility testing is only one stage in the cycle of developing complex interventions [43]. 
Table 5 Health state values and QALYS

\begin{tabular}{|c|c|c|c|c|c|c|c|c|c|c|}
\hline & \multicolumn{5}{|c|}{ Usual care } & \multicolumn{5}{|l|}{ BRiMS } \\
\hline & Mean & (SD) & Min & Max & $N$ & Mean & (SD) & Min & Max & $N$ \\
\hline \multicolumn{11}{|c|}{ Baseline data: } \\
\hline EQ5D-3L & 0.58 & $(0.16)$ & 0.04 & 0.77 & 26 & 0.54 & $(0.17)$ & -0.04 & 0.88 & 29 \\
\hline EQ5D-5L & 0.66 & $(0.20)$ & 0.07 & 0.89 & 26 & 0.63 & $(0.17)$ & 0.22 & 0.95 & 29 \\
\hline MSIS-8D & 0.51 & $(0.21)$ & 0.08 & 0.80 & 26 & 0.49 & $(0.15)$ & 0.21 & 0.76 & 30 \\
\hline \multicolumn{11}{|c|}{ Week 15 data: } \\
\hline EQ5D-3L & 0.60 & $(0.18)$ & 0.20 & 0.91 & 24 & 0.59 & $(0.17)$ & -0.00 & 0.88 & 25 \\
\hline EQ5D-5L & 0.69 & $(0.18)$ & 0.19 & 0.95 & 24 & 0.67 & $(0.17)$ & 0.26 & 0.95 & 25 \\
\hline MSIS-8D & 0.54 & $(0.20)$ & 0.13 & 0.82 & 24 & 0.56 & $(0.16)$ & 0.22 & 0.83 & 25 \\
\hline \multicolumn{11}{|c|}{ Week 27 data: } \\
\hline EQ5D-3L & 0.59 & $(0.25)$ & -0.13 & 0.91 & 22 & 0.57 & $(0.11)$ & 0.30 & 0.77 & 22 \\
\hline EQ5D-5L & 0.67 & $(0.25)$ & 0.05 & 0.95 & 22 & 0.65 & $(0.15)$ & 0.38 & 0.89 & 22 \\
\hline MSIS-8D & 0.56 & $(0.19)$ & 0.08 & 0.88 & 22 & 0.54 & $(0.17)$ & 0.18 & 0.77 & 22 \\
\hline \multicolumn{11}{|c|}{ Estimated QALYs (over 27 weeks): } \\
\hline EQ5D-3L & 0.30 & $(0.08)$ & 0.13 & 0.43 & 22 & 0.30 & $(0.05)$ & 0.20 & 0.42 & 22 \\
\hline EQ5D-5L & 0.34 & $(0.09)$ & 0.11 & 0.46 & 22 & 0.34 & $(0.07)$ & 0.22 & 0.47 & 22 \\
\hline MSIS-8D & 0.28 & $(0.10)$ & 0.09 & 0.42 & 22 & 0.29 & $(0.06)$ & 0.20 & 0.40 & 22 \\
\hline
\end{tabular}

\section{Participant characteristics}

In recognition of the prevalence of comorbidity in MS, our recruitment criteria set out to be as inclusive as possible, and the sample characteristics were in line with publications in this field [44]. Whilst the allocation between groups was similar for most MS-related characteristics, the differing distribution of some (such as EDSS, anxiety/depression and cognition) could potentially affect outcomes, as evidenced by differences in baseline measures between the groups (e.g. MSWS-12v2, MiniBEST). This suggests that randomisation in a future definitive trial may require stratification by these characteristics, and that potential subgroup analyses should be considered when developing an a priori statistical analysis plan. The baseline characteristics also highlight that our sample was more severely balance and mobility impaired in comparison to a number of other studies with similar sample EDSS levels [21, 45]. For example, Gijbels et al. [46] report mean walking distances of 104 metres in the two minute walking test in a sample of 21 people with an EDSS between 4.5-6.5. In comparison, on average, our sample walked around $53 \mathrm{~m}$ at baseline. In addition, our sample reported higher levels of concern (as measured by the FESi) than other MS populations of mixed MS subtypes, although this is perhaps not surprising given their falls history and progressive MS [47].

\section{Proposed primary outcome measure}

A key aim was to obtain data to inform the selection of a primary outcome measure for the definitive trial. The major consequence of falling for the individual is increasing mobility impairment, activity curtailment and loss of confidence $[8,48,49]$. Therefore, based on the existing evidence base, together with the results from this feasibility study, we recommend that the primary outcome for a definitive trial is the MSWS-12v2 [16]. Whilst a direct measure of injurious falls would be our favoured option given its clinical importance, our hesitancy in recommending this outcome is the recognised issues with the validity and reliability of falls diary data $[50,51]$, as evidenced by the problems with data completeness and accuracy we also experienced. If these issues can be resolved, then injurious falls should be re-considered as a primary outcome in a definitive trial. However, it is recognised that this would likely require a significantly larger sample size.

\section{Health economics data}

Methods for collection of data (costs, outcomes) for a future economic evaluation were feasible and few challenges were faced in relation to this. The results highlight the relatively modest resource use of 'formal' health and care resources by the study participants, and the high use of 'informal' care and support. Our study is unable to determine if this pattern is through necessity (e.g. due to lack of resources) or choice; however, the findings reflect the importance of collecting comprehensive resource use data capturing both formal and informal care and support. The estimated health state values and QALY estimates are lower when using the MSIS$8 \mathrm{D}$, and further research is recommended to consider why this may be (for example, being linked to specific domains of health-related QoL that may not be covered 
fully by the EQ-5D). However, the MSIS-8D indicates potential to show differences between groups over time.

\section{Strengths and limitations}

A key strength of this feasibility study is that it used robust methodology, with comprehensive step-by-step documentation and evaluation of our processes, decisions and outcomes. However, there were some limitations. Most notably, despite adhering to best-practice recommendations [15] and with previous high return rates using similar methods $[5,52]$, the low return rate of the self-report paper-based falls diaries means that our falls data must be interpreted with caution. Our results highlight the need to find a valid and reliable method of collecting these data before falls can be considered as a potential primary outcome. Further, our assumption that if participants did not return a fall diary they did not fall, errs on the side of underestimating falls. In addition, the participants were only followed up for 3 months, and hence operational issues (such as study retention) and clinical outcomes are unknown for a longer follow-up period.

\section{Conclusions and recommendations}

This study assessed the feasibility of undertaking a definitive trial to compare BRiMS plus usual care to usual care alone in a sample of people with SPMS who reported themselves as falling. We have demonstrated the study procedures to be feasible. Retention, programme engagement and outcome completion rates were all sufficient to satisfy our a priori progression criteria. Challenges were experienced in some areas, such as the completion of daily self-report fall diaries. A future trial should consider alternative methods of collecting these data. Estimated sample sizes for a definitive trial with MSWS-12v2 as the primary outcome range from 575 to 990 participants.

\section{Supplementary Information}

The online version contains supplementary material available at https://doi. org/10.1186/s40814-020-00732-9.

Additional file 1. Summary statistics, mean, standard deviation (SD) and range, and between-group differences of the potential secondary outcome measures $[15,17,20,23,24,27,28,53-63]$.

Additional file 2. Potential secondary outcome analyses [46, 56, 64-66].

Additional file 3. Indicative sample sizes for a definitive trial of BRiMS

Additional file 4. Health and social care/informal care costs [67].

\section{Acknowledgements}

The authors acknowledge the following:

- NIHR HTA Programme for providing funding for the study;

- NHS trusts and clinical research networks who supported recruitment and ran the programmes;
- Staff of the Peninsula Clinical Trials unit who supported the day-to-day running of the trial;

- Members of the Trial management group, TSC and Data monitoring committee for contributing their time and expertise; and

Patient volunteers who contributed their time and energy to participating in the study and providing feedback.

\section{Authors' contributions}

$J F, H G, J A, L P, L R, S C, C G, P E, J V, A B$ and $B M$ designed the overall study. SC and $\mathrm{KS}$ were the trial statisticians and were responsible for writing the full statistical analysis plan and undertaking the analyses. $\mathrm{HG}, J \mathrm{~J}, \mathrm{MB}, \mathrm{CG}$, JV and SC wrote the manuscript. HG, JF, MB, JV, LP, LR, SC, PE and CG wrote the study protocol. JZ provided input to the study design. All authors critically proof read and approved the final manuscript.

\section{Availability of data and materials}

Individual participant data that underlie the results of this study will be made available (following de-identification) on a controlled access basis, subject to suitable data sharing agreements. Requests for data sharing should be made to the Chief Investigator (Cl, Freeman) in the first instance. Requesters will be asked to complete an application form detailing specific requirements, rationale and proposed usage. Requests will be reviewed by the $\mathrm{Cl}$ and study Sponsor who will consider the viability and suitability of the request and the credentials of the requester. Where access to requested data is granted, requesters will be asked to sign a data sharing agreement. Requested data will be made available, along with supporting documentation (e.g. data dictionary) on a secure server or via other secure data transfer method.

\section{Ethics approval and consent to participate}

The study protocol, participant information and enrolment procedures were assessed and approved through the National Research Ethics Scheme (NRES Committee South West -Exeter REC ref no. 16/SW/0266) and the NHS Health Research Authority (HRA) (ref IRAS 199153). Any amendments to the protocol were reported to, and approved via NRES, and the HRA.

Consent for publication

Not applicable

\section{Competing interests}

The authors declare that they have no competing interests. This study was funded by the NIHR Health Technology Assessment Programme (14/176/12), United Kingdom. This article presents independent research. The views expressed are those of the authors and not necessarily those of the NHS, the NIHR or the Department of Health. The sponsor of this clinical study was the Plymouth Hospitals NHS Trust, Devon, England, UK.

\section{Author details}

${ }^{1}$ Faculty of Health, School of Health Professions, Peninsula Allied Health Centre, University of Plymouth, Derriford Road, Plymouth PL6 8BH, England. ${ }^{2}$ Faculty of Health, Medical Statistics Group, Room N15, Plymouth Science Park, Plymouth PL6 8BX, England. ${ }^{3}$ Peninsula Clinical Trials Unit, University of Plymouth, Room N16, Plymouth Science Park, Plymouth PL6 8BX, England. ${ }^{4}$ University of Exeter Medical School, College of Medicine \& Health, University of Exeter, Exeter, England. ${ }^{5}$ Faculty of Health, School of Psychology, University of Plymouth, Portland Square Building, Drake Circus Campus, Plymouth PL4 8AA, England. ${ }^{6}$ School of Health \& Life Sciences, Glasgow Caledonian University, Cowcaddens Road, Glasgow G4 OBA, Scotland. ${ }^{7}$ Douglas Grant Rehabilitation Unit, Ayrshire Central Hospital, Kilwinning Road, Irvine KA12 8SS, Scotland. ${ }^{8}$ University of Exeter Medical School, Health Economics Group, University of Exeter, St. Luke's Campus, Exeter EX1 2LU, England. ${ }^{9} \mathrm{NIHR}$ Research Design Service (South West), Musgrove Park Hospital, Taunton TA1 5DA, England. ${ }^{10}$ Faculty of Medicine and Dentistry, NIHR Research Design Service South West, ITTC Building, Plymouth Science Park, Plymouth PL6 8BX, England. ${ }^{11}$ School of Medicine, Medical and Biological Sciences, University of St. Andrews, North Haugh, St. Andrews KY16 9TF, Scotland. 
Received: 14 June 2019 Accepted: 23 November 2020 Published online: 04 January 2021

\section{References}

1. Royal College of Physicians. The national audit of services for people with multiple sclerosis 2011. London: Royal College of Physicians; 2011.

2. Giesser BS. Primer on multiple sclerosis. New York: Oxford University Press; 2016.

3. Cattaneo D, Jonsdottir J, Zocchi M, Regola A. Effects of balance exercises on people with multiple sclerosis: a pilot study. Clin Rehabil. 2007;21(9):771-81.

4. Soyuer F, Mirza M, Erkorkmaz U. Balance performance in three forms of multiple sclerosis. Neurol Res. 2006;28(5):555-62.

5. Gunn H, Creanor S, Haas B, Marsden JF, Freeman J. Risk factors for falls in Multiple Sclerosis: an Observational Study. Mult Scler. 2013;19(14):1913-22.

6. Feinstein A, Freeman J, Lo AC. Treatment of progressive multiple sclerosis: what works, what does not, and what is needed. Lancet Neurol. 2015;14(2): 194-207.

7. Gunn H, Markevics S, Haas B, Marsden J, Freeman J. Systematic Review: The Effectiveness of Interventions to Reduce Falls and Improve Balance in Adults With Multiple Sclerosis. Arch Phys Med Rehabil. 2015;96(10):1898-912.

8. Gunn H, Endacott R, Haas B, Marsden J, Freeman J. Development of a balance, safe mobility and falls management programme for people with multiple sclerosis. Disabil Rehabil. 2017;40(24):1-10.

9. Gunn H, Andrade J, Paul L, Miller L, Creanor S, Green C, et al. Balance Right in Multiple Sclerosis (BRiMS): a guided self-management programme to reduce falls and improve quality of life, balance and mobility in people with secondary progressive multiple sclerosis: a protocol for a feasibility randomised controlled t. Pilot Feasibility Stud. 2018;4(1):26.

10. Multiple Sclerosis Society UK. Relapsing Remitting MS (RRMS) [Internet]. 2016 [cited 2016 Dec 21];Available from: https://www.mssociety.org.uk/ what-is-ms/types-of-ms/relapsing-remitting-rrms.

11. Gunn H, Andrade J, Paul L, Creanor S, Stevens K, Green C, et al. A guided self-management programme to reduce falls and improve quality of life, balance and mobility in people with secondary progressive Multiple Sclerosis: The Balance Right in Multiple Sclerosis (BRiMS) feasibility randomised controlled trial. 2018.

12. Multiple Sclerosis Society. A lottery of treatment and care - MS services across the UK. London: Multiple Sclerosis Society; 2013.

13. Carter A, Daley A, Humphreys L, Snowdon N, Woodroofe N, Petty J, et al. Pragmatic intervention for increasing self-directed exercise behaviour and improving important health outcomes in people with multiple sclerosis: a randomised controlled trial. Mult Scler. 2014;20(8):1112-22.

14. Thomas S, Thomas PW, Kersten P, Jones R, Green C, Nock A, et al. A pragmatic parallel arm multi-centre randomised controlled trial to assess the effectiveness and cost-effectiveness of a group-based fatigue management programme (FACETS) for people with multiple sclerosis. J Neurol Neurosurg Psychiatry. 2013;84(10):1092-9.

15. Coote S, Sosnoff JJ, Gunn H. Fall Incidence as the Primary Outcome in Multiple Sclerosis Falls-Prevention Trials. Int J MS Care. 2014;16(4):178-84.

16. Hobart J, Cano S. Improving the evaluation of therapeutic interventions in multiple sclerosis: The role of new psychometric methods. Health Technol Assess (Rockv). 2009;13(12):iii ix-x, 1-177.

17. Herdman M, Gudex C, Lloyd A, Janssen M, Kind P, Parkin D, et al. Development and preliminary testing of the new five-level version of EQ-5D (EQ-5D-5L). Qual Life Res. 2011;20(10):1727-36.

18. Hobart JC. The Multiple Sclerosis Impact Scale (MSIS-29). In: Jenkinson C, Peters M, Bromberg M, editors. Quality of Life Measurement in Neurodegenerative and Related Conditions. Cambridge: Cambridge University Press; 2011. p. 24-40

19. Hawton A, Green C, Telford C, Zajicek J, Wright D. Using the Multiple Sclerosis Impact Scale to Estimate Health State Utility Values: Mapping from the MSIS-29, Version 2, to the EQ-5D and the SF-6D. Value Heal. 2012;15(8): 1084-91.

20. Grant PM, Ryan CG, Tigbe WW, Granat MH. The validation of a novel activity monitor in the measurement of posture and motion during everyday activities. Br J Sports Med. 2006;40(12):992-7.

21. Gijbels D, Eijnde BO, Feys P. Comparison of the 2- and 6-minute walk test in multiple sclerosis. Mult Scler. 2011;17(10):1269-72.

22. Franchignoni F, Horak F, Godi M, Nardone A, Giordano A. Using psychometric techniques to improve the Balance Evaluation Systems Test: the mini-BESTest. J Rehabil Med. 2010;42(4):323-31.
23. Yardley L, Beyer N, Hauer K, Kempen G, Piot-Ziegler C, Todd C. Development and initial validation of the Falls Efficacy Scale-International (FES-I). Age Ageing. 2005;34(6):614-9.

24. Heinemann AW, Magasi S, Bode RK, Hammel J, Whiteneck GG, Bogner J, et al. Measuring enfranchisement: Importance of and control over participation by people with disabilities. Arch Phys Med Rehabil. 2013;94(11): 2157-65.

25. R Core Team. R: A language and environment for statistical computing. 2019.

26. Eldridge SM, Chan CL, Campbell MJ, Bond CM, Hopewell S, Thabane L, et al. CONSORT 2010 statement: extension to randomised pilot and feasibility trials. Pilot Feasibility Stud. 2016;2(1):64.

27. Hobart JCJ, Riazi A, Lamping DLD, Fitzpatrick R, Thompson AJA. Measuring the impact of MS on walking ability - The 12-Item MS Walking Scale (MSWS-12). Neurology. 2003;60(1):31-6.

28. Hobart J, Lamping D, Fitzpatrick R, Riazi A, Thompson A. The Multiple Sclerosis Impact Scale (MSIS-29): a new patient-based outcome measure. Brain. 2001;124(Pt 5):962-73.

29. Delbaere $K$, Close JCT, Mikolaizak AS, Sachdev PS, Brodaty H, Lord SR. The Falls Efficacy Scale International (FES-I). A comprehensive longitudinal validation study. Age Ageing. 2010;39(2):210-6.

30. van Hout B, Janssen MF, Feng Y-S, Kohlmann T, Busschbach J, Golicki D, et al. Interim Scoring for the EQ-5D-5L: Mapping the EQ-5D-5L to EQ-5D-3L Value Sets. Value Heal. 2012;15(5):708-15.

31. Goodwin E, Green C, Spencer A. Estimating a Preference-Based Index for an Eight-Dimensional Health State Classification System for Multiple Sclerosis. Value Heal. 2015;18(8):1025-36.

32. National Institute for Health and Care Excellence (NICE). Position statement on use of the EQ-5D-5L valuation set. London: National Institute for Health and Care Excellence; 2017. p. 4-6.

33. Mehta L, McNeill M, Hobart J, Wyrwich KW, Poon J-L, Auguste P, et al. Identifying an important change estimate for the Multiple Sclerosis Walking Scale-12 (MSWS-12v1) for interpreting clinical trial results. Mult Scler J - Exp Transl Clin. 2015;1:205521731559699.

34. Kohn CG, Sidovar MF, Kaur K, Zhu Y, Coleman Cl. Estimating a minimal clinically important difference for the EuroQol 5-Dimension health status index in persons with multiple sclerosis. Health Qual Life Outcomes. 2014; 12:66.

35. Costelloe L, O'Rourke K, Kearney H, McGuigan C, Gribbin L, Duggan M, et al. The patient knows best: significant change in the physical component of the Multiple Sclerosis Impact Scale (MSIS-29 physical). J Neurol Neurosurg Psychiatry. 2007;78(8):841-4.

36. Pals SL, Murray DM, Alfano CM, Shadish WR, Hannan PJ, Baker WL. Individually randomized group treatment trials: a critical appraisal of frequently used design and analytic approaches. Am J Public Health. 2008; 98(8):1418-24.

37. Roberts C, Roberts SA. Design and analysis of clinical trials with clustering effects due to treatment. Clin Trials J Soc Clin Trials. 2005;2(2):152-62.

38. Motl RW, Dlugonski D, Pilutti LA, Klaren RE. Does the effect of a physical activity behavioral intervention vary by characteristics of people with multiple sclerosis? Int J MS Care. 2015;17(2):65-72.

39. Nilsagård $Y$, Westerdahl E, Wittrin A, Gunnarsson M. Walking distance as a predictor of falls in people with multiple sclerosis. Physiother Res Int. 2016; 21(2):102-8

40. Zajicek JP, Ingram WM, Vickery J, Creanor S, Wright DE, Hobart JC. Patientorientated longitudinal study of multiple sclerosis in south west England (The South West Impact of Multiple Sclerosis Project, SWIMS) 1: protocol and baseline characteristics of cohort. BMC Neurol. 2010;10(1):88.

41. Carter A, Humphreys L, Snowdon N, Sharrack B, Daley A, Petty J, et al. Participant recruitment into a randomised controlled trial of exercise therapy for people with multiple sclerosis. Trials. 2015;16(1):468.

42. Pilutti LA, Platta ME, Motl RW, Latimer-Cheung AE. The safety of exercise training in multiple sclerosis: a systematic review. J Neurol Sci. 2014;343(1-2):3-7.

43. Campbell NC, Murray E, Darbyshire J, Emery J, Farmer A, Griffiths F, et al. Designing and evaluating complex interventions to improve health care. BMJ. 2007:334(7591):455-9.

44. Marrie RA, Cohen J, Stuve O, Trojano M, Sørensen PS, Reingold S, et al. A systematic review of the incidence and prevalence of comorbidity in multiple sclerosis: overview. Mult Scler. 2015;21(3):263-81.

45. Langeskov-Christensen D, Feys P, Baert I, Riemenschneider M, Stenager E, Dalgas $U$. Performed and perceived walking ability in relation to the 
Expanded Disability Status Scale in persons with multiple sclerosis. J Neurol Sci. 2017;382:131-6.

46. Gijbels D, Alders G, Van Hoof E, Charlier C, Roelants M, Broekmans T, et al. Predicting habitual walking performance in multiple sclerosis: relevance of capacity and self-report measures. Mult Scler J. 2010;16(5):618-26.

47. van Vliet R, Hoang P, Lord S, Gandevia S, Delbaere K. The Falls Efficacy Scale International: A Cross-Sectional Validation in People with Multiple Sclerosis. Arch Phys Med Rehabil. 2013;94(5):883-9.

48. Gunn H, Creanor S, Haas B, Marsden J, Freeman J. Frequency, characteristics and consequences of falls in multiple sclerosis: findings from a cohort study. Arch Phys Med Rehabil. 2014;95(2):538-45.

49. Peterson EW, Cho CC, Finlayson ML. Fear of falling and associated activity curtailment among middle aged and older adults with multiple sclerosis. Mult Scler. 2007;13(9):1168-75.

50. Hunter H, Rochester L, Morris R, Lord S. Longitudinal falls data in Parkinson's disease: feasibility of fall diaries and effect of attrition. Disabil Rehabil. 2018; 40(19):2236-41.

51. Griffin J, Lall R, Bruce J, Withers E, Finnegan S, Lamb SE, et al. Comparison of alternative falls data collection methods in the Prevention of Falls Injury Trial (PreFIT). J Clin Epidemiol. 2019;106:32-40.

52. Hoang PD, Baysan M, Gunn H, Cameron M, Freeman J, Nitz J, et al. Fall risk in people with MS: A Physiological Profile Assessment study. Mult Scler J Exp Transl Clin. 2016;2(0):1898-912

53. National Institute for Health and Care Excellence. Guide to the methods of technology appraisal. London: NICE; 2013.

54. Lamb S, Jørstad-Stein E, Hauer K, Becker C. Development of a common outcome data set for fall injury prevention trials: the prevention of Falls Network Europe Consensus. J Am Geriatr Soc. 2005;9:1618-22.

55. Gijbels D, Dalgas U, Romberg A, De Groot V, Bethoux F, Vaney C, et al. Which walking capacity tests to use in multiple sclerosis? A multicentre study providing the basis for a core set. Mult Scler J. 2012;18(3):364-71.

56. Godi M, Franchignoni F, Caligari M, Giordano A, Turcato AM, Nardone A. Comparison of Reliability, Validity, and Responsiveness of the Mini-BESTest and Berg Balance Scale in patients with balance disorders. Phys Ther. 2012;93(2):158-67.

57. Tyson S, Connell L. The psychometric properties and clinical utility of measures of walking and mobility in neurological conditions: a systematic review. Clin Rehabil. 2009;23(11):1018-33.

58. Freeman JA, Gear M, Pauli A, Cowan P, Finnigan C, Hunter $\mathrm{H}$, et al. The effect of core stability training on balance and mobility in ambulant individuals with multiple sclerosis: a multi-centre series of single case studies. Mult Scler J. 2010;16(11):1377-84.

59. Paltamaa J, Sjögren T, Peurala SH, Heinonen A. Effects of physiotherapy interventions on balance in multiple sclerosis: a systematic review and metaanalysis of randomized controlled trials. J Rehabil Med. 2012;44(10):811-23.

60. Finlayson M, Peterson E, Patricia N. Participation as an Outcome in MS Fall Prevention Research: Consensus Recommendations from the International MS Fall Prevention Research Network. Int J MS Care. 2014;16(4):171-77.

61. Green C, Richards DA, Hill JJ, Gask L, Lovell K, Chew-Graham C, et al. Costeffectiveness of collaborative care for depression in UK primary care: Economic evaluation of a randomised controlled trial (CADET). PLOS ONE. 2014;9(8): e104225.

62. Goodwin E, Green C. Improving the measurement of QALYs in multiple sclerosis: Estimating a preference-based index for use in deriving qualityadjusted life-years (QALYS) for multiple sclerosis. Qual Life Res. 2014;23(15).

63. Goodwin E, Green C, Brazier JEE, Tsuchiya A, Dolan P, Brazier JEE, et al. A quality-adjusted life-year measure for multiple sclerosis: developing a patient-reported health state classification system for a multiple sclerosisspecific preference-based measure. Value Heal. 2015;18(8):1016-24.

64. Connelly DM, Thomas BK, Cliffe SJ, Perry WM, Smith RE. Clinical utility of the 2-minute walk test for older adults living in long-term care. Physiother Can. 2009;61(2):78-87.

65. Mann GC, Whitney SL, Redfern MS, Borello-France DF, Furman JM. Functional reach and single leg stance in patients with peripheral vestibular disorders. J Vestib Res. 1996;6(5):343-53.

66. Morgan MT, Friscia LA, Whitney SL, Furman JM, Sparto PJ. Reliability and validity of the Falls Efficacy Scale-International (FES-I) in individuals with dizziness and imbalance. Otol Neurotol. 2013;34(6):1104-8.

67. Curtis L, Burns A. Unit costs of health and social care. Kent: University of Kent; 2016.

\section{Publisher's Note}

Springer Nature remains neutral with regard to jurisdictional claims in published maps and institutional affiliations.

\section{Ready to submit your research? Choose BMC and benefit from:}

- fast, convenient online submission

- thorough peer review by experienced researchers in your field

- rapid publication on acceptance

- support for research data, including large and complex data types

- gold Open Access which fosters wider collaboration and increased citations

- maximum visibility for your research: over $100 \mathrm{M}$ website views per year

At BMC, research is always in progress.

Learn more biomedcentral.com/submissions 